\title{
The contribution of GLONASS measurements to regional and continental scale geodetic monitoring regimes
}

\author{
M. P. Stewart ${ }^{1}$, M. Tsakiri ${ }^{1}$, J. Wang ${ }^{1}$, and J. F. Monico ${ }^{2}$ \\ ${ }^{1}$ Curtin University of Technology, Western Australia \\ ${ }^{2}$ FCT/UNESP, Brazil \\ (Received December 6, 1999; Revised May 12, 2000; Accepted May 15, 2000)
}

\begin{abstract}
Throughout late 1998 and early 1999, the International GLONASS Experiment (IGEX) has delivered the first comprehensive inter-continental dual frequency GLONASS data set. This experiment represents the first opportunity to assess how a second global satellite positioning system could complement existing GPS geodetic infrastructure. Based on analysis of a three station network of IGEX stations from Southern Hemisphere IGEX stations, this paper discusses the internal and external precision of long baseline GPS, GLONASS and combined GPS/GLONASS solutions, and the possible contribution of GLONASS to future regional-scale geodetic work.
\end{abstract}

\section{Introduction}

The tracking of GPS and GLONASS satellites as part of the International GLONASS Experiment (IGEX) officially commenced on the 20th October. For the duration of the experiment, over 60 GPS/GLONASS receivers have been located at sites around the world. Run under the auspices of the International Association of Geodesy (IAG), the International GPS Service (IGS), the International Earth Rotation Service (IERS) and the Institute of Navigation (ION), this experiment represents the first opportunity to assess how a second global satellite positioning system could complement existing GPS infrastructure for geodetic purposes (Willis and Slater, 1999).

At the time of writing (November 1999), the GLONASS constellation has been reduced to 10 operating satellites, less than half the full constellation of 24 . With the current economic situation in the Russian Federation and the aged status of the remaining constellation, the future of the GLONASS system is looking increasingly uncertain. The launch of three new GLONASS satellites in December 1998, and their subsequent coming on line in January/February 1999, led to a peak in the number of available satellites in the early months of the year. If the status of the GLONASS system continues to decline and with no alternative global positioning systems becoming available in the near future, the data collected during the IGEX campaign in the early months of 1999 will represent a unique data set for several years to come. Over this period, a sufficient number of GLONASS satellites (generally greater than half the constellation) were operational to allow studies of the likely contribution secondary satellite positioning systems could make to well established GPS-based global and regional monitoring regimes.

The availability of a second GPS-like global positioning

Copy right $(\mathrm{C}$ The Society of Geomagnetism and Earth, Planetary and Space Sciences (SGEPSS); The Seismological Society of Japan; The Volcanological Society of Japan; The Geodetic Society of Japan; The Japanese Society for Planetary Sciences. system would have two significant benefits to geodetic monitoring. First, solutions from a second system could provide an independent check on GPS solutions, thus improving quality control. Second, raw observations from the second system could be combined directly with raw GPS observations to provide a geometrically stronger solution (GPS is known to be geometrically weaker in height and at high latitudes).

In this paper, we illustrate results from a subset of the southern hemisphere network of IGEX stations described in Stewart et al. (1999), in the context of the comparison of stand-alone GPS and GLONASS solutions, and solutions from direct combination of raw GPS/GLONASS observations.

\section{Regional Observation Network}

The sub-network comprises three stations, Yarragadee and Mt. Stromlo, both located in Australia, and Crary Science Laboratory, McMurdo, Antarctica. The Australian stations used Ashtech Z18 dual frequency receivers whilst a Javad Positioning Systems Legacy dual frequency receiver was installed at Crary. Figure 1 shows the regional sub-network described in this paper. Baseline lengths range from 3,199 km (Yarragadee-Mt. Stromlo) to 5,558 km (Yarragadee-Crary).

Each of the three baselines was processed using a separate week of data. Allocation of one baseline per week ensured that separate baseline solutions were independent. Therefore, the baseline network could be subsequently subjected to a least squares network adjustment without considering baseline correlations. Solutions were computed on a daily basis and then combined into multi-arc solutions using the CODE precise GPS and GLONASS orbits computed by the Centre for Orbit Determination (CODE), Berne (Ineichen et al., 1999). GPS-only, GLONASS-only and combined GPS/GLONASS solutions were computed. Due to limitations on the length of this paper, the reader is referred to Stewart et al. (1999) for full details of the basic processing methodology for the GPS-only and GLONASS- 


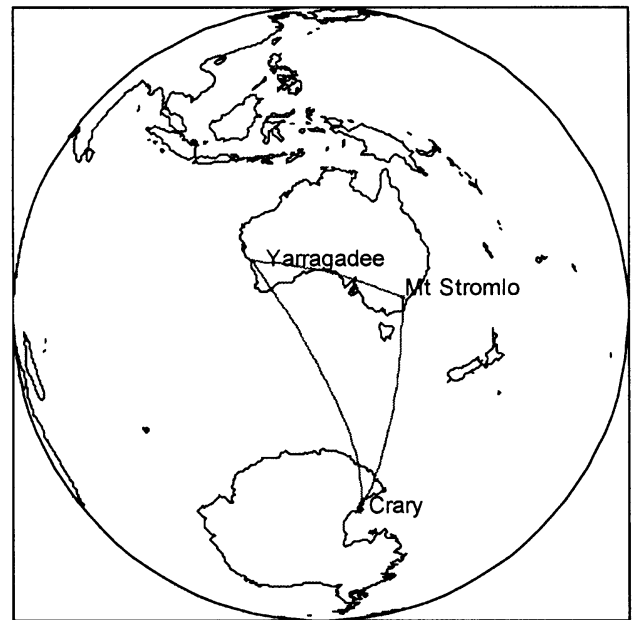

Fig. 1. Southern hemisphere region sub-network.

only solutions.

\section{Combined GPS/GLONASS Solutions}

Raw GPS and GLONASS carrier phase observations are fundamentally similar, with the main difference being that whilst GPS satellites transmit on the same L-band frequencies, GLONASS satellites transmit on different, albeit similar, frequencies. Therefore, the double differenced observable formed from raw GLONASS observations still contains some clock biases (e.g. Raby and Daly, 1994). One solution to this problem is to scale the GLONASS L1 and L2 observations from each satellite to a common frequency (e.g. Leick, 1998). In the data processing presented in this paper, GLONASS L1 and L2 carrier phase observations were scaled to the GPS L1 and L2 frequencies respectively.

Once scaled to the GPS L1 and L2 frequencies, GLONASS carrier phase observations, in terms of data processing, may be treated as if they were GPS observations, with three major qualifiers. First, the integer nature of the GLONASS double difference ambiguity is lost. However, as the ionospheric free linear combination of L1 and L2 for GPS also results in a situation where ambiguities and cycle slips are non-integer in nature, on long baselines this factor is not an issue. Second, a difference exists between the reference frame in which the GLONASS satellite orbits are computed and the GPS satellite orbits are computed. Finally, a time offset between GPS and GLONASS time systems must be accounted for.

Point three above has by far the most critical effect when directly combining GPS and GLONASS observations. Without the availability of highly accurate values for the time offset between the two systems, it is necessary to sacrifice an observation to solve for this offset. In the processing described here, only GPS-GPS and GLONASS-GLONASS double difference observations were formed in the combined solution, thus negating between system time biases.

\section{Results}

Table 1 gives the individual statistics for the three multi-arc baseline solutions. It can be seen that the number of double difference satellite observations increases by between $20 \%$ and $40 \%$ when GLONASS is included in the full solution. The estimated noise on the double difference observations is increased marginally for the combined solution, though not sufficiently to indicate that GLONASS observations are significantly noisier than GPS observations. The associated precision of the individual baseline components $\left(\sigma_{\Delta x}, \sigma_{\Delta y}, \sigma_{\Delta z}\right)$ generally decreases, indicating improved geometric strength in the solution when the additional GLONASS observations are included. Note that these statistics are only internal precision indicators i.e. they are derived from the final solution covariance matrix. It is generally accepted that GPS covariance matrices tend to derive error estimates that are too optimistic and the error estimates for the GPS, GLONASS and combined GPS/GLONASS solutions presented here are no exception. These precisions are presented rather to demonstate the improvement in geometric strength of the combined GPS/GLONASS solutions over the individual GPS and GLONASS solutions. Baseline lengths are in agreement for all three type of solution at the $0.01 \mathrm{ppm}$ level, demonstrating that even with an incomplete constellation, GLONASS-only solutions are comparable to GPS. The higher uncertainty on the baseline components from the GLONASS-only solutions represents the lower number of double difference GLONASS observations that could be formed in comparison to GPS.

It is also evident from Table 1 that the internal precision of the baseline from Mt. Stromlo to Crary is worse than the other two lines. This is attributed to a lower number of GPS observations available over the processed multi-day arc for that baseline. In GPS week 0992, the Z18 receiver at Stromlo recorded only between 12 and 16 hours of data per day. The outage did not reduce the number of GLONASS observations available, however (in fact, more GLONASS measurements were observed in total on this line than Yarragadee-Crary). It is also possible that this additional noise is caused by more inclement antarctic atmospheric conditions in week 992, although further analysis would be required to justify this statement. Baseline Yarragadee to Stromlo was processed for week 991 during which no such outage problems occurred.

As each baseline is independent of the other, a more realistic estimate of solution precision may be derived from computing a three dimensional least squares fit of vector components of the three baselines. This provides an external precision check, rather than relying on the over-optimistic standard deviations given in Table 1 . Table 2 shows the formal errors of the estimated station coordinates at Crary and Mt. Stromlo at the $1 \sigma$ confidence level from a 3-dimensional least squares adjustment of the three independent baselines with Yarragadee held fixed. It is interesting to note that the standard deviations from the combined solution are greater than those from either the GPS-only or GLONASS-only solutions, contrary to the improvement in internal precision shown by the standard deviations on the individual baseline components. It would appear that whilst the GPS-only and GLONASS-only baseline solutions yield similar baseline lengths, the orientation of the baseline vectors agree less well. The orientation of the baseline vector solutions is controlled through the reference frame defined by the fixed satellite orbits. Therefore, this result implies differences in the reference frames defined by GPS and GLONASS fixed orbits. It may be speculated that as the CODE IGEX GLONASS or- 
Table 1. Baseline statistics from GPS, GLONASS and GPS/GLONASS processing.

\begin{tabular}{ccccccc}
\hline \multicolumn{7}{c}{ Yarragadee-Stromlo } \\
\hline solution type & No. obs. & $\sigma_{\Delta x}(\mathrm{~m})$ & $\sigma_{\Delta y}(\mathrm{~m})$ & $\sigma_{\Delta z}(\mathrm{~m})$ & obs. noise & Length $(\mathrm{m})$ \\
\hline GPS & 6391 & 0.009 & 0.010 & 0.007 & $4.3 \mathrm{~mm}$ & 3199303.737 \\
GLONASS & 2005 & 0.015 & 0.025 & 0.016 & $4.6 \mathrm{~mm}$ & 3199303.736 \\
GPS + GLONASS & 8396 & 0.005 & 0.007 & 0.005 & $4.7 \mathrm{~mm}$ & 3199303.695 \\
\hline Nolution type & No. obs. & $\sigma_{\Delta x}(\mathrm{~m})$ & $\sigma_{\Delta y}(\mathrm{~m})$ & $\sigma_{\Delta z}(\mathrm{~m})$ & obs. noise & Length $(\mathrm{m})$ \\
\hline GPS & 5896 & 0.006 & 0.010 & 0.011 & $5.5 \mathrm{~mm}$ & 5778302.223 \\
GLONASS & 1034 & 0.020 & 0.039 & 0.042 & $5.5 \mathrm{~mm}$ & 5778302.177 \\
GPS + GLONASS & 6930 & 0.004 & 0.009 & 0.010 & $6.4 \mathrm{~mm}$ & 5778302.204 \\
\hline solution type & No. obs. & $\sigma_{\Delta x}(\mathrm{~m})$ & $\sigma_{\Delta y}(\mathrm{~m})$ & $\sigma_{\Delta z}(\mathrm{~m})$ & obs. noise & Length $(\mathrm{m})$ \\
\hline GPS & 3628 & 0.014 & 0.007 & 0.018 & $6.7 \mathrm{~mm}$ & 4698509.709 \\
GLONASS & 1783 & 0.035 & 0.023 & 0.029 & $5.0 \mathrm{~mm}$ & 4698509.730 \\
GPS + GLONASS & 5411 & 0.023 & 0.011 & 0.027 & $7.0 \mathrm{~mm}$ & 4698509.700 \\
\hline
\end{tabular}

Table 2. Standard deviations from least squares network adjustment from GPS-only, GLONASS-only and GPS/GLONASS solutions.

\begin{tabular}{|c|c|c|c|c|c|c|c|c|c|}
\hline Station & $\sigma_{x}(\mathrm{~m})$ & $\sigma_{y}(\mathrm{~m})$ & $\sigma_{z}(\mathrm{~m})$ & $\sigma_{x}(\mathrm{~m})$ & $\sigma_{y}(\mathrm{~m})$ & $\sigma_{z}(\mathrm{~m})$ & $\sigma_{x}(\mathrm{~m})$ & $\sigma_{y}(\mathrm{~m})$ & $\sigma_{z}(\mathrm{~m})$ \\
\hline & \multicolumn{3}{|c|}{ GPS } & \multicolumn{3}{|c|}{ GLONASS } & \multicolumn{3}{|c|}{ GPS/GLONASS } \\
\hline Crary & 0.015 & 0.020 & 0.031 & 0.021 & 0.028 & 0.028 & 0.036 & 0.046 & 0.061 \\
\hline Mt. Stromlo & 0.002 & 0.013 & 0.022 & 0.015 & 0.021 & 0.015 & 0.028 & 0.038 & 0.026 \\
\hline
\end{tabular}

bits used for the analysis presented here are restricted to a smaller number of tracking stations than in the northern hemisphere, reference frame definition for the IGEX GLONASS orbits may be more uncertain in the Southern Hemisphere.

\section{Concluding Remarks}

The main difficulty with processing data from the IGEX experiment on baselines of several thousand kilometres in length is the lack of available GLONASS satellites with which to form double difference observations. Taking into account that only fixed precise ephemerides and basic modelling techniques were applied, results from the adjusted GLONASS-only solution are encouraging. It seems likely that the larger error ellipses in the GLONASS least squares solution are due to the availability of fewer GLONASS observations. GLONASS solutions demonstrate similar characteristics to GPS and, if fully operational, could be used as an independent alternative to GPS for geodetic purposes.

Even with a restricted GLONASS constellation, baseline lengths from the two positioning systems have been shown to agree at the $0.01 \mathrm{ppm}$ level. Although other space geodetic techniques can be used for independent validation of regional GPS networks, in practical terms, only GLONASS (or similar future systems) could provide additional information at each monitoring points. GPS and GLONASS solutions may never be completely independent (for example, in the IGEX campaign observations were taken by the same receiver to the same antenna) but regional solutions from two separate systems could provide an indication of systematic biases, in a field where very small changes in station coordinates are often interpreted as tectonic motion.

The internal precision (i.e. the geometrical strength) of long baseline solutions were improved when combined GPS/GLONASS solutions were performed. Furthermore, the baseline lengths from the GPS-only, GLONASS-only and combined solutions were consistent. However, the least squares adjustment of the three independent baselines from the combined solution yielded larger error ellipses than for either the GPS-only or GLONASS-only solutions. In addition, the estimated observation noise was higher on each baseline for the combined solution. As on baselines of several thousand kilometres in length orbital error is significant, it is likely that these results are a direct result of the GLONASS satellite orbits being less well determined (in terms of reference frame and/or precision) that the GPS orbits. It may be concluded that if GPS and GLONASS observations from long baselines are to be combined directly into one solution using fixed GLONASS precise orbits computed from 
the existing IGEX network, some form of downweighting is necessary for the GLONASS observations to account for GLONASS orbital uncertainties. Conversely, it may be anticipated that this problem could be eliminated if (or when) GLONASS precise orbital computation approaches a similar level of resolution as GPS precise orbits.

Acknowledgments. The authors would like to thank the reviewers for some constructive comments regarding an earlier draft of this paper.

\section{References}

Ineichen, D., M. Rothatcher, T. Springer, and G. Beutler, Results of CODE as an Analysis Centre of the IGEX-98 Campaign, IGEX-99 Workshop, September 13-14, Nashville, Tennessee, U.S.A., 1999.
Leick, A., GLONASS satellite surveying, Journal of Surveying Engineering, 121, 91-99, 1998.

Raby, P. and P. Daly, Surveying with GLONASS: Calibration, Error Sources and Results, Proceedings 3rd International Symposium on Differential Satellite Navigation Systems, Canary Wharf, London, U.K., April 1822, 1994.

Stewart, M. P., M. Tsakiri, J. Wang, and J. F. Monico, IGEX-A regional analysis of data from the Southern Hemisphere, Proceedings of the IGEX98 Workshop, Nashville, U.S.A., September 13-14, 1999.

Willis, P. and J. Slater, International GLONASS Experiment (IGEX-98), in IGS 1998 Annual Report, pp. 400-838, Jet Propulsion Laboratory, California Institute of Technology, Pasadena, California, JPL Publication, 1999.

M. P. Stewart(e-mail: stewart@vesta.curtin.edu.au), M. Tsakiri, J. Wang, and J. F. Monico 